\title{
Investigating Thermal Behavior of Surface Phonon in SiC by in-situ Vibrational Spectroscopy
}

\author{
Xingxu Yan ${ }^{1}$, Chaitanya A. Gadre ${ }^{2}$, Sheng Dai ${ }^{1}$, Kehang Yu ${ }^{1}$, Xiaoqing Pan $^{1,2}$ \\ 1. Department of Chemical Engineering and Materials Science, University of California - Irvine, Irvine, \\ California 92697 \\ 2. Department of Physics and Astronomy, University of California - Irvine, Irvine, California 92697
}

Monochromated electron energy-loss spectroscopy (EELS) has the capability of detecting vibrational spectrum of materials with energy resolution of sub-10 meV. Utilizing such powerful technique in dedicated scanning transmission electron microscope (STEM), recent studies have focused on the phonon modes in various types of materials, including beam-sensitive materials [1], organic softmaterials [2], insulating oxides [3] and two-dimensional materials [4]. Combined with high resolution of STEM, spatial distribution of both bulk and surface phonon modes can be revealed in nanometer scale [3]. The vibrational spectrum in EELS also needs special interpretation whose treatment is different from traditional optical approaches [4]. Furthermore, temperature-dependence of phonon modes can provide more fundamental physical information such as thermal expansion of crystal, and atomic motion at high temperature. Here, we investigate thermal behavior of surface phonon polariton $(\mathrm{SPhP})$ in $\mathrm{SiC}$ film by in-situ vibrational spectroscopy.

$\mathrm{SiC}$ is a wide-bandgap semiconductor with high rigidity, superior chemical inertness, large thermal conductivity and a low thermal expansion coefficient, and widely used in power electronics. Hence, we choose $\mathrm{SiC}$ as a good system to study thermal behavior of phonon mode. In our experiment, SiC film is the heating substrate of Protochips Fusion ${ }^{\mathrm{TM}}$ thermal chip, which is compatible with Nion UltraSTEM's cartridge design. The temperature of $\mathrm{SiC}$ film are set from room temperature to $1000{ }^{\circ} \mathrm{C}$, controlled by commercial Protochips software. Vibrational spectra are obtained by Nion UltraSTEM 200 at University of California, Irvine, equipped with $\mathrm{C} 3 / \mathrm{C} 5$ aberration corrector and high-energy resolution monochromated EELS system (HERMES). The instrument is operated at an accelerating voltage of $60 \mathrm{kV}$ with probe convergence and collection semi-angles of 30 and $10 \mathrm{mrad}$, respectively. Each EELS measurement consists of 200 frames with 1s exposure time of each frame, which are aligned by the center of zero loss peak (ZLP), for better signal intensity. Then, background subtraction is performed by fitting an exponential function [3].

Fig. 1a shows HAADF-STEM image on edge of SiC film with 3C phase. All vibrational spectra are acquired at the red point, which is $20 \mathrm{~nm}$ away from the sample in aloof configuration [1]. Fig. 1b and c are background subtracted spectra of both energy gain and energy loss region under various temperatures. At room temperature $(298 \mathrm{~K})$, only a singular peak is visible in the energy loss region located at about $116 \mathrm{meV}$. The peak is assigned as SPhP mode in SiC [5]. However, no bulk mode (transversal optical mode of $\sim 100 \mathrm{meV}$ or longitudinal optical mode of $\sim 120 \mathrm{meV}$ ), was observed in loof configuration. This result indicates that only SPhP mode can be excited when the probe is outside the sample. With increasing temperature, the peak shifts toward lower energy region. When temperature increases, the lattice spacing is widened and the force constant is reduced, causing the phonon energy to decrease. Interestingly, an extra peak appears at the energy gain region, when the temperature is over $473 \mathrm{~K}$, and its intensity becomes much stronger with increasing of temperature. Fig. 2a summarizes the peak centers of both energy loss and gain peaks by Gaussian fitting. The error bar of energy gain peak 
center is worse than that of energy loss one due to the weaker peak intensity. The peak positions in the gain and loss regime are equidistant from the ZLP and both shift closer to the ZLP with increasing temperature. At higher temperatures, more phonons are excited transferring energy to electron beam. Phonon peaks in the gain regime are similar to anti-Stokes peaks in Raman spectroscopy. Furthermore, the energy shift is proportional to the change of temperature with the slope of $-5.5 \mathrm{meV} / 1000 \mathrm{~K}$. Fig. 2b plots the peak center of simulated phonon spectra under various temperature molecular dynamic (MD) calculation. The simulated phonon peak shift to low energy region with a slope of $-4.9 \mathrm{meV} / 1000 \mathrm{~K}$. Considering both lattice expansion and atom motion at high temperature can fully explain our experimental data.

\section{References:}

[1] O. L. Krivanek et al, Nature 514 (2014), p. 209-212.

[2] P. Rez et al, Nat. Commun. 7 (2016), 10945.

[3] M. J. Lagos, A. Trugler, U. Hohenester, and P. E. Batson, Nature 543, (2017), p. 529-532.

[4] A. A. Govyadinov et al., Nat. Commun. 8 (2017), 95.

[5] M. Dayan, Surf. Sci. 149 (1985), p. L33-L38.

[6] The authors acknowledge the support of the University of California Irvine Materials Research Institute for the use of TEM facilities.
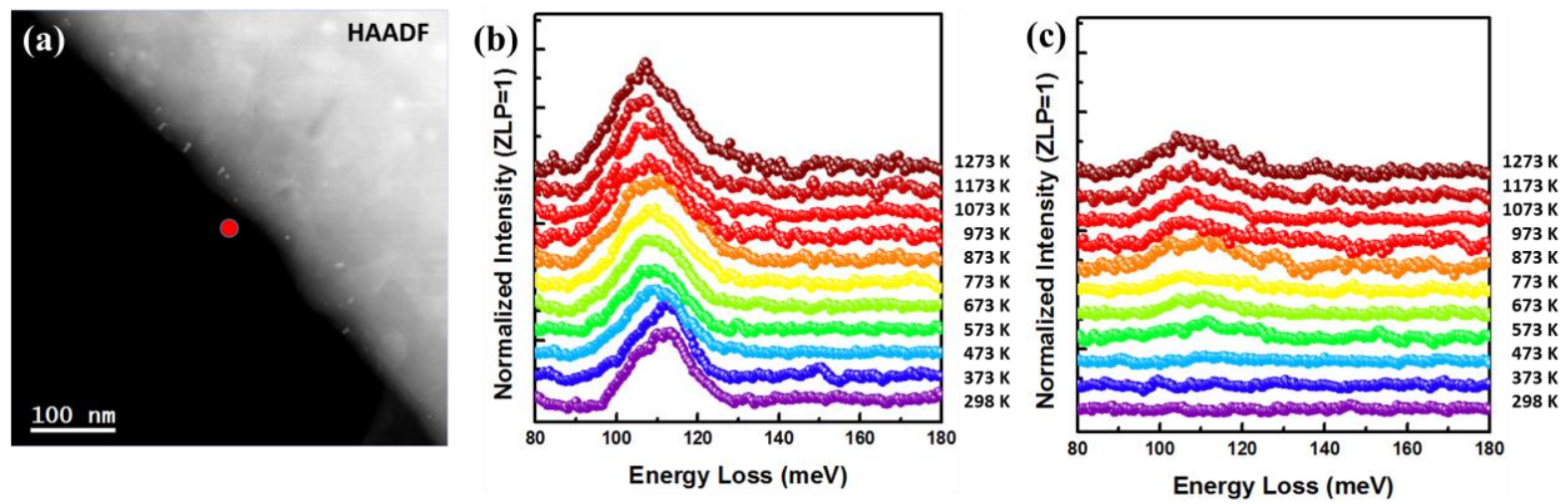

Figure 1. (a) HAADF-STEM image of SiC film. The red point is the location we acquired vibrational spectra in aloof configuration. (b) Energy loss and (c) Energy gain regions of vibrational spectra under different temperature. All spectra have been removed the background. The temperatures are indexed near the curves.
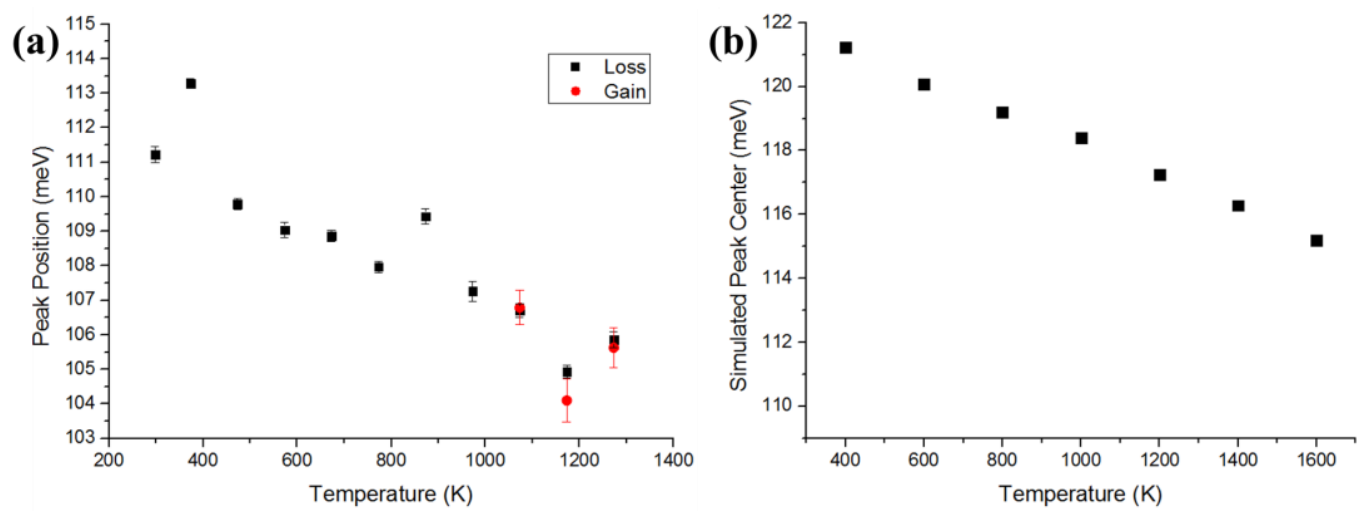

Figure 2. (a) Dependence of energy loss and gain (only three high temperatures) peak centers with the temperature. (b) Simulated peak center of phono peak at different temperature. 